\title{
Spacetimes with continuous linear isotropies III: null rotations
}

\section{A. H. MacCallum ${ }^{1}$}

Received: 20 June 2021 / Accepted: 11 October 2021 / Published online: 25 October 2021 (c) The Author(s) 2021

\begin{abstract}
It is shown that in many of the possible cases local null rotation invariance of the curvature and its first derivatives is sufficient to ensure that there is an isometry group $G_{r}$ with $r \geq 3$ acting on (a neighbourhood of) the spacetime and containing a null rotation isotropy. The exceptions where invariance of the second derivatives is additionally required to ensure this conclusion are Petrov type $\mathrm{N}$ Einstein spacetimes, spacetimes containing "pure radiation" (a Ricci tensor of Segre type [(11,2)]), and conformally flat spacetimes with a Ricci tensor of Segre type $[1(11,1)]$ (a "tachyon fluid").
\end{abstract}

\section{Contents}

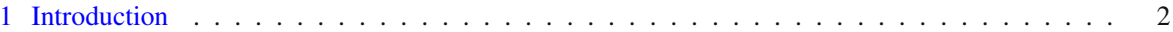

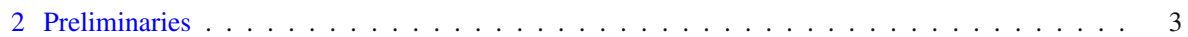

2.1 Canonical forms of the curvature tensor, and frame choices . . . . . . . . . . . 3

2.1 .1 The Weyl tensor . . . . . . . . . . . . . . . . . . . . . . . . . . . . . . . . . . .

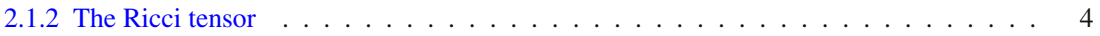

2.1.3 Possible LNRI groups and further specializations . . . . . . . . . . . . . . . . . . . 5

2.1.4 Implications for the Cartan-Karlhede procedure . . . . . . . . . . . . . . . . . . . 6

2.1 .5 Additional invariants . . . . . . . . . . . . . . . . . . . . . . . . 7

2.2 LNRI and derivatives of $\Psi \ldots \ldots \ldots \ldots \ldots \ldots \ldots$

2.3 The Bianchi identities . . . . . . . . . . . . . . . . . . . . . . . . . . . . . . . . . . . . . . . .

2.4 LNRI and derivatives of $\Phi \ldots \ldots \ldots \ldots \ldots \ldots \ldots$

3 LNRI spacetimes . . . . . . . . . . . . . . . . . . . . . . . . 10

3.1 LNRI spacetimes with $\Phi_{A B^{\prime}}=0 \ldots \ldots \ldots \ldots \ldots \ldots \ldots \ldots$

3.2 LNRI spacetimes with a Ricci tensor of Segre type $[(1,3)] \ldots \ldots$. . . . . . . . . . . . . . . 13

3.3 LNRI spacetimes with a Ricci tensor of Segre type $[1(1,2)] \ldots$. . . . . . . . . . . . . . . . 13

3.4 Petrov N LNRI spacetimes with a Ricci tensor of Segre type $[(11,2)]$. . . . . . . . . . . . . 14

3.5 Conformally flat LNRI spacetimes with a Ricci tensor of Segre type $[(11,2)] \ldots \ldots$

3.6 LNRI spacetimes with a Ricci tensor of Segre type $[1(11,1)] \ldots \ldots$. . . . . . . . . . . . 16

4 Conclusion . . . . . . . . . . . . . . . . . . . . . . . 16

A Known spacetimes with null rotation invariance . . . . . . . . . . . . . . . . . . . . . 17

$\otimes$ M. A. H. MacCallum

M.A.H.MacCallum@qmul.ac.uk

1 School of Mathematical Sciences, Queen Mary University of London, Mile End Road, London, UK 
B The Bianchi identities under LNRI . . . . . . . . . . . . . . . . . . . . . . . . . 21

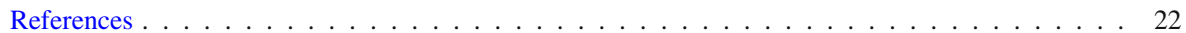

\section{Introduction}

This paper studies local null rotation invariance in spacetime, using the same definitions, techniques, conventions and notation as the companion papers studying local spatial rotation invariance and local boost invariance [11,12], i.e. the Cartan-Karlhede procedure implemented using the Newman-Penrose (NP) formalism, the software CLASSI and the minimal set of totally symmetric spinors defined by [13]. Those details will, for brevity, not be repeated here. As in the previous papers, the "NewmanPenrose equations" (the Ricci equations) and Bianchi identities [(7.21a)-(7.21r) and (7.32a)-(7.32k) respectively in [19]] will be referred to below as (NPa)-(NPr) and (Ba)-(Bk). The original NP notation $\Lambda=R / 24$ will be used, rather than the Ricci scalar $R$, and a prime on $\Phi_{A B^{\prime}}$ (denoted $\Phi_{A B}$ in [19]) is retained for consistency with the compressed notation for symmetrized spinors representing higher derivatives. Note that the Newman-Penrose $\Lambda$ and the cosmological constant (typically also denoted by $\Lambda$ ) are not the same. Denoting the cosmological constant by $L$ for clarity, $L=R / 4$.

Following [3], $\left(\mathrm{A}_{m}\right)$ is the assumption that

At each point $P$ in an open neighbourhood $U$ of a point $P_{o}$, there exists a nondiscrete subgroup $g$ of the Lorentz group in the tangent space $T_{P}$ which leaves invariant the curvature tensor and all its covariant derivatives to the $m$-th order,

Here, as in the previous papers, the focus is on finding the minimal $m$ for a given $g$ which ensures that Ellis's definition (C) holds, i.e.

There exists a local group of motions $G_{r}$ in an open neighbourhood $W$ of a point $P_{o}$ which is multiply transitive on some $q$ [-dimensional] surface through each point $P$ of $W$.

The $G_{r}$ will then include, at each $P \in W$, an isotropy subgroup isomorphic to $g$ leaving $P$ fixed. (In some cases there may in addition be a proper homothety: see Sections 8.7 and 11.3 of [19].)

In this paper the group $g$ is assumed to contain a group of null rotations ${ }^{1}$. This will be referred to as local null rotation invariance, which will be abbreviated to LNRI. For clarity the term 'local null rotation isotropy' will be used only where (C) applies and the $G_{r}$ contains, for each $P \in W$, an isometry fixing $P$ which is a null rotation. This paper aims to find the minimal values of $m$ in $\left(\mathrm{A}_{m}\right)$ for which LNRI implies local null rotation isotropy.

A one-parameter group of null rotations preserves a null direction and an orthogonal spacelike direction. Any directions invariantly defined by the curvature tensor must be of those characters for LNRI to apply. The only Weyl tensors satisfying this condition

\footnotetext{
${ }^{1}$ Here this term denotes one of the transformations (3.14) or (3.15) of [19], rather than the more general combination of these with a boost and rotation given the same name in [4]. Note that in (3.15) of [19] the final $\boldsymbol{l}$ should be $\boldsymbol{k}$.
} 
are either zero (conformally flat) or of Petrov type N. The possible Ricci tensors are discussed in Sect. 2.1.2.

Section 2 sets out the choices of frame and canonical form for the Weyl and Ricci curvatures in Sect. 2.1, and their possible implications, and then in the following subsections, respectively the conditions arising from LNRI of a Petrov type N Weyl tensor, from the Bianchi identities, and from LNRI of $\Phi_{A B^{\prime}}$. In Sect. 3, the minimal $\left(\mathrm{A}_{m}\right)$ guaranteeing null rotation isotropy in the different possible types of LNRI spacetimes are studied. Other than spacetimes with constant curvature, these are Petrov type $\mathrm{N}$ spacetimes with $\Phi_{A B^{\prime}}=0$ and Petrov type $\mathrm{N}$ and conformally flat spacetimes with non-zero $\Phi_{A B^{\prime}}$ of certain Segre types. The appendices summarize the known spacetimes admitting LNRI and the form of the Bianchi identities with the specializations of Sect. 2.1.

The results of Sect. 3 lead to:

Theorem 1 Einstein spacetimes with a Weyl tensor of Petrov type N, spacetimes with a Ricci tensor of Segre type $[(11,2)]$, and conformally flat spacetimes with a Ricci tensor of Segre type [1(11,1)], are locally null rotation isotropic if and only if their curvature tensor and its first two derivatives are locally null rotation invariant.

Note that the statement remains true for those subcases of the spacetime families covered by the theorem for which LNRI of the first derivatives implies LNRI of the second derivatives. The specification of those subcases is given in Sects. 3.1, 3.4, 3.5 and 3.6.

Theorem 2 A spacetime other than those covered by Theorem 1 is locally null rotation isotropic if and only if the curvature tensor and its first derivative are locally null rotation invariant.

\section{Preliminaries}

\subsection{Canonical forms of the curvature tensor, and frame choices}

\subsubsection{The Weyl tensor}

In Petrov type N, the Weyl tensor is invariant under a two-dimensional group of null rotations. A suitable canonical form, if the repeated principal null direction preserved by the null rotations is $\boldsymbol{k}$ as will be assumed from here on ${ }^{2}$, is one in which a nonzero Weyl spinor has only $\Psi_{4} \neq 0$.

In terms of an aligned spinor basis, the group of null rotations fixing $\boldsymbol{k}$ is

$$
o^{\star}=o, \quad \iota^{\star}=\iota+B o,
$$

where $\star$ denotes the transformed values and $B$ is a complex number. If there is invariance of the curvature and its derivatives for all values of $B$ in (2.1), the LNRI group is

\footnotetext{
2 As in the previous papers, choosing specific frames is for computational convenience. The results, being invariantly expressed, would be the same in any frame, though they might be harder to check.
} 
two-dimensional. The transforms of the curvature spinors and their derivatives will be polynomials in $B$ and $\bar{B}$ with the untransformed values as coefficients, rather than just multiples of the untransformed values as in spatial rotation or boost invariance. The test for LNRI then involves a set of linear equations in components of the curvature and its derivatives, as in [10], rather than just the vanishing of some components.

Applying a position-dependent boost, $\left|\Psi_{4}\right|$ will be set to 1 in Petrov type N. A position-dependent spatial rotation will then be used to make $\Psi_{4}$ constant: the remaining frame freedom now consists of a constant spatial rotation and position-dependent null rotation(s) about $\boldsymbol{k}$. The spatial rotation freedom could be used to set $\Psi_{4}=1$, but when there is only a one-parameter LNRI group it is more convenient to use it to make the parameter $B$ in (2.1) pure imaginary.

That is possible, assuming that the one-parameter LNRI group acts in the same way at all points in the neighbourhood $U$, because in that case $B=|B| e^{i \theta}$ with a fixed $\theta$ and varying $|B|$. The remaining rotation freedom can be used to set $\theta=\pi / 2$. (The rotation that does this will not in general also give $\Psi_{4}=1$.) With this one-parameter LNRI group there is still the freedom of a null rotation with position-dependent $|B|$.

In conformally flat spaces $\Psi_{4}=0$ and the Weyl tensor gives no information on frame choice. $\Lambda$ also cannot restrict the frame. The tracefree part of the Ricci tensor, $\Phi_{A B^{\prime}}$, remains to be considered.

\subsubsection{The Ricci tensor}

As stated above, for a Ricci tensor to have LNRI, any invariant directions it defines must either be null or in spacelike directions orthogonal to the null direction invariant under the LNRI. In the following the invariant direction is assumed to be the $\boldsymbol{k}$ of a Newman-Penrose frame adapted to the curvature.

As shown in [10], a totally symmetrized spinor $\chi_{a b^{\prime}}$ of valence $(m, n)$ with $m \geq n$, such as one of the minimal set of Cartan invariants defined in [13], is invariant under (2.1) only if ${ }^{3}$

$$
\begin{aligned}
\chi_{a b^{\prime}} & =0 \quad \text { for all pairs } a+b \leq m-1 \\
0 & =(a+1) B \chi_{a b^{\prime}}+b \bar{B} \chi_{(a+1)(b-1)^{\prime}} \text { otherwise. }
\end{aligned}
$$

(Here one can exchange the roles of the pair $m, a$ with those of $n, b$.) For a twoparameter LNRI group the only non-zero component is $\chi_{m n^{\prime}}$. Note that the conditions for a one-parameter LNRI group still apply (trivially) in that case.

Assuming a one-parameter LNRI group with $B$ imaginary, (2.3) becomes

$$
(a+1) \chi_{a b^{\prime}}=b \chi_{(a+1)(b-1)^{\prime}} .
$$

For LNRI of the Ricci curvature, $\Phi_{A B^{\prime}}$ must be invariant under (2.1). The Segre types of the possible such Ricci tensors are listed in Table 1, taken from Table 5.2 of [19].

\footnotetext{
3 The example found in [14] which requires the 7th derivative of the Riemann tensor to complete the Cartan-Karlhede procedure is a Petrov type $\mathrm{N}$ spacetime which has no isotropies. However, it obeys the conditions (2.2)-(2.3) at steps 0 and 1 of the Cartan-Karlhede procedure (Åman, private communication).
} 
Table 1 Nontrivial invariance groups containing null rotations, by Ricci tensor type

\begin{tabular}{ll}
\hline Invariance group & Segre type of the Ricci tensor \\
\hline One-parameter group of null rotations & {$[1(1,2)],[(1,3)]$} \\
Null rotations and spatial rotations & {$[(11,2)]$} \\
$S O(2,1):$ three-dimensional Lorentz group & {$[1(11,1)]$} \\
Full Lorentz group & {$[(111,1)]$} \\
\hline
\end{tabular}

Segre type $[(111,1)]$ implies that $\Phi_{A B^{\prime}}=0$ for all $A$ and $B$, so the spacetime is an Einstein space. The Bianchi identities (see Appendix B) then imply that $\Lambda$ is constant. If such a spacetime is conformally flat, it is one of the well-known constant curvature spacetimes, which need not be considered further $\left(\left(\mathrm{A}_{0}\right)\right.$ with $s_{0}=6$ is sufficient in this case to ensure (C) above). Petrov type N Einstein spaces are included in Sect. 3.1.

Among the other types admitting a possible null rotation invariance, only type $[(11,2)]$ is interpretable in general relativity in terms of one of the standard models of macroscopic matter. It represents "pure radiation", such as a null electromagnetic field. Type $[1(11,1)]$ can represent a tachyonic fluid, and type $[1(1,2)]$ could be considered a superposition of pure radiation and a tachyonic fluid. but neither is generally regarded as occurring in nature. Type [(1,3)] violates the usual energy conditions. For these reasons types $[1(11,1)],[1(1,2)]$ and $[(1,3)]$ were not considered in [19]. They are retained here for completeness. With any $\Phi_{A B^{\prime}}$, there could be a nonzero $\Lambda$.

Applying (2.2)-(2.3), one finds from (2.2) that $\Phi_{00^{\prime}}=\Phi_{01^{\prime}}=0$ and, from (2.3) with imaginary $B$, that $\Phi_{02^{\prime}}=2 \Phi_{11^{\prime}}$ if $\Phi_{11^{\prime}} \neq 0$, and $\Phi_{12^{\prime}}$ is real if nonzero. When $\Phi_{12^{\prime}} \neq 0=\Phi_{11}$, a position-dependent null rotation can be used to bring the curvature to a canonical form in which $\Phi_{22^{\prime}}=0$. Otherwise $\Phi_{22^{\prime}} \neq 0$ is possible. These considerations lead to the following canonical forms for the Segre types compatible with an at least one-dimensional LNRI group, expressed in an NP tetrad with $\boldsymbol{k}$ as the null direction preserved by the null rotations. The nonzero components $\Phi_{A B^{\prime}}$ are:

Segre type [(1,3)]: $\Phi_{12^{\prime}} \neq 0$ is real.

Segre type [1(1,2)]: $\Phi_{02^{\prime}}=2 \Phi_{11^{\prime}} \neq 0 \neq \Phi_{22^{\prime}}$.

Segre type [(11,2)]: $\Phi_{22^{\prime}} \neq 0$.

Segre type $[1(11,1)]: \Phi_{02^{\prime}}=2 \Phi_{11^{\prime}} \neq 0$.

The last two cases are specializations of the canonical form for Segre type [1(1,2)] and there are thus strong correspondences between the calculations for these three cases.

\subsubsection{Possible LNRI groups and further specializations}

In conformally flat spacetimes, the null direction $\boldsymbol{k}$ defining the LNRI group cannot be fixed by the (zero) Weyl tensor, and must be determined using the Ricci tensor and its derivatives. As discussed above Ricci tensors of Segre type $[(111,1)]$ give only spaces of constant curvature and Petrov type N Einstein spaces. A Ricci tensor of Segre type $[1(11,1)]$ is invariant under null rotations about either of two directions, say $\boldsymbol{k}$ and $\boldsymbol{l}$, but if both these are in $g$, so is a boost invariance in the $(\boldsymbol{k}, \boldsymbol{l})$ plane because there 
Fig. 1 Possible pairs $(s, t)$ and their evolution in successive steps of the Cartan-Karlhede procedure

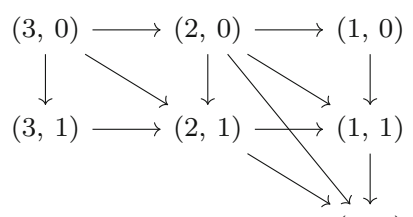

$(1,2)$

is no two-parameter subgroup of the Lorentz group generated by such a pair of null rotations (see Table 6.1 of [4]). Such boost invariant spacetimes are conformally flat, have $s=3$, admit a $G_{6}$ or $G_{7}$ and were considered in [12]. Hence for Segre type $[1(11,1)]$ only spacetimes which are LNRI about $\boldsymbol{k}$ and not $\boldsymbol{l}$ need be considered here. The remaining possible Segre types define the $\boldsymbol{k}$ direction uniquely.

The canonical forms above have been chosen so that for those spacetimes admitting only a one-dimensional LNRI group, $B$ in (2.1) is imaginary. Having fixed the $\boldsymbol{k}$ direction one may still apply a spatial rotation and/or boost preserving it. A Ricci tensor of Segre type $[(11,2)]$ has spatial rotation invariance, but the boost can be used in the conformally flat case to set $\left|\Phi_{22^{\prime}}\right|=1$, and this can similarly be achieved in type $[1(1,2)]$. In conformally flat spacetimes with a Ricci tensor of Segre type $[(1,3)]$ one can set $\Phi_{12^{\prime}}=1$, using the boost and spatial rotation freedoms (a position-dependent rotation was used to make $B$ imaginary but the remaining constant rotation freedom can be used to make the necessarily real $\Phi_{12^{\prime}}$ positive).

One may note that with these choices of canonical frame in a conformally flat spacetime the left side of each of the Bianchi identities is real, so that the imaginary part of each right side must vanish. Moreover, if $t_{0}=0$ all components of the curvature are constants and therefore all the left sides of the Bianchi identities are zero. The deductions from Bianchi and Ricci identities for conformally flat spacetimes with Ricci tensors of Segre types [(11,2)] and [1(11,1)] considered in, respectively, [11] and [12] remain true in the LNRI cases.

\subsubsection{Implications for the Cartan-Karlhede procedure}

The figure in [11] which shows possible evolution of pairs $\left(s_{i}, t_{i}\right)$ at step $i$ of the CartanKarlhede procedure, is repeated here as Figure 1: for full explanation see Section 2.2 of [11].

Ricci tensors of types [(11,2)] and [1(11,1)] are invariant under a three-parameter linear isotropy group, so conformally flat spaces with such Ricci tensors have as their $\left(s_{0}, t_{0}\right)$ pair one of the $(3, T)$ pairs in Fig. 1. However, as discussed above, spacetimes with a Ricci tensor of type $[1(11,1)]$ either have a boost invariance and were covered in [12] or have only a one-dimensional LNRI group. Similarly spacetimes with a Ricci tensor of type $[(11,2)]$ which have a spatial rotation invariance were discussed in [11]. So in this paper only groups $g$ consisting solely of null rotations need be considered.

All Petrov type $\mathrm{N}$ spacetimes with LNRI have $s_{0}=2$ or 1 and a $g$ consisting only of null rotations. For LNRI conformally flat spacetimes the group $g$ of null rotations can be two-dimensional if $\Phi_{A B^{\prime}}=0$, which would only give only the constant curvature spacetimes, or if $\Phi_{A B^{\prime}}$ is of Segre type [(11,2)]. Otherwise $s=s_{1}=1$, so that $\left(s_{1}, t_{1}\right)$ is of the form $(1, T)$ where $T$ represents an allowed value of $t$ in Fig. 1. 
If $Q$ is a scalar function of position, then the vector defined by $Q_{, a}$ is

$$
Q_{, a}\left(2 m^{(a} \bar{m}^{b)}-2 k^{(a} l^{b)}\right)=\delta Q \overline{\boldsymbol{m}}+\bar{\delta} Q \boldsymbol{m}-D Q \boldsymbol{l}-\Delta Q \boldsymbol{k},
$$

and if $Q$ is invariantly defined this must be a linear combination of vectors invariant under the LNRI group. The number of such independent vectors bounds $t_{i}$ above.

If there is a two-dimensional LNRI group, $\boldsymbol{k}$ defines the only direction invariant under $g$, but if the LNRI group is one-dimensional there are two invariant directions, the second being given by $B \overline{\boldsymbol{m}}-\bar{B} \boldsymbol{m}$, which for imaginary $B$ is in the direction $\boldsymbol{m}+\overline{\boldsymbol{m}}$. Whether $s=1$ or $2,(2.5)$ shows that for any LNRI scalar $Q, D Q=0$, and if $s=2$, $\delta Q=\bar{\delta} Q=0$ also. If $s=1$ and $B$ is imaginary, $\delta Q=\bar{\delta} Q$, and this is real for real $Q$. So in either case one will have, for an invariant $Q$,

$$
D Q=0, \quad \delta Q=\bar{\delta} Q
$$

where $\delta Q$ will be real if $Q$ is, and will be zero if $s=2$.

In the Cartan-Karlhede procedure, this implies that if $s_{i+1}=2, t_{i} \leq 1$. So when $s=2$ the Cartan-Karlhede procedure terminates at the latest at $p=2$ (if the $(s, t)$ sequence is $(3,0) \rightarrow(2,0) \rightarrow(2,1) \rightarrow(2,1))$ and thus $\left(\mathrm{A}_{3}\right)$ suffices. If for all $i \geq 0, s_{i+1}=1=s$, then $t_{i} \leq 2$. In principle $\left(\mathrm{A}_{4}\right)$ could now be required, for a sequence starting at $\left(s_{0}, 0\right)$ for some $s_{0}>1$ and then running through each of the $(1, T)$ possibilities in Figure 1 (so $t_{0}=t_{1}=0$ ). However, this could only happen in Petrov type N Einstein spacetimes, type N “pure radiation” spacetimes, or conformally flat spacetimes with Ricci tensors of Segre types [(11,2)] or [1(11,1)]. Otherwise, $\left(\mathrm{A}_{3}\right)$ would again suffice. In the sequel it is found that in all those cases $\left(\mathrm{A}_{4}\right)$ is satisfied if $\left(\mathrm{A}_{0}\right)-\left(\mathrm{A}_{3}\right)$ are, and no new information arises at the fourth derivative.

With a $g$ consisting solely of null rotations, if $s=2$ then $t_{p} \leq 1$ and there will be an isometry group $G_{r}$ with $r \geq 5$. If $s=1$ there is an isometry group $G_{3}$ with $r \geq 3$. Appendix A reviews known spacetime metrics consistent with these bounds.

After studying the possible combinations of Weyl and Ricci tensors, it turned out to be more convenient to consider the conformally flat and Petrov $\mathrm{N}$ cases together in most Segre types with $\Phi_{A B^{\prime}} \neq 0$, using the Bianchi identities and the conditions imposed on $\Phi$ by LNRI before those arising from LNRI of $\Psi$. The exception is Segre type $[(11,2)]$ where the calculations for the Petrov $\mathrm{N}$ and conformally flat cases have fewer common features.

\subsubsection{Additional invariants}

The directional derivative conditions above for LNRI apply not only to components of the curvature and its derivatives in the canonically-chosen frames, but also to those spin coefficients, or combinations thereof, completely determined by the frame specializations. Several cases below lead to

$$
\kappa=\rho=\sigma=\varepsilon=0, \quad \alpha=\beta+\tau,
$$


and if that is true the following are invariant under the remaining frame freedom: $\alpha$, $\beta, \tau$, and, when $B$ is imaginary, $\pi+\bar{\pi}$. When (2.7) holds, if $\alpha=0$ or if there is a one-parameter LNRI group with imaginary $B$, then $\gamma$ is also null rotation invariant. For brevity such null rotation invariants will often be called just invariants in this paper.

Two more invariant combinations of spin coefficients are found in the cases studied below. For a one-parameter LNRI group with imaginary $B, \lambda+\bar{\mu}$ is invariant if $\kappa+\bar{\kappa}=0=\rho+\bar{\sigma}$ and

$$
\pi+\bar{\pi}+2(\alpha-\bar{\beta})=0 .
$$

Under the same conditions, $v+\bar{v}$ is invariant if $\varepsilon$ is real,

$$
(\tau+\bar{\tau})+(\pi+\bar{\pi})=0,
$$

and

$$
\lambda+\bar{\mu}+2 \bar{\gamma}=2 \gamma+\bar{\lambda}+\mu
$$

(i.e. $\lambda+\bar{\mu}+2 \bar{\gamma}$ is real). (The invariants' properties $D Q=0=(\delta-\bar{\delta}) Q$ can usually be obtained from the NP equations also, but this has not been checked in all cases.)

\subsection{LNRI and derivatives of $\Psi$}

In a canonical frame chosen as above, all directional derivatives of $\Psi_{4}$ vanish and $\nabla \Psi_{A B^{\prime}}$ has as possibly non-zero components

$$
\begin{aligned}
& \nabla \Psi_{30^{\prime}}=2 \kappa \Psi_{4} / 5, \quad \nabla \Psi_{31^{\prime}}=2 \sigma \Psi_{4} / 5, \\
& \nabla \Psi_{40^{\prime}}=\left[4(\varepsilon+\rho) \Psi_{4}\right] / 5, \\
& \nabla \Psi_{41^{\prime}}=\left[4(\beta+\tau) \Psi_{4}\right] / 5, \\
& \nabla \Psi_{50^{\prime}}=4 \alpha \Psi_{4}, \quad \nabla \Psi_{51^{\prime}}=4 \gamma \Psi_{4} .
\end{aligned}
$$

Whether the LNRI group has $s=1$ or 2 , if $\Psi_{4} \neq 0$, (2.2) and (2.3) imply $\nabla \Psi_{30^{\prime}}=$ $\nabla \Psi_{31^{\prime}}=\nabla \Psi_{40^{\prime}}=0$, so that

$$
\kappa=\sigma=\varepsilon+\rho=0 .
$$

The Ricci tensor has not yet been specified, but one may note that the null rotation invariance ensures that condition $(\mathrm{C})$ of the Kundt-Thompson theorem as stated in [19] is satisfied, and also that when $\rho=0$ these spacetimes are members of Kundt's class (Chapter 31 in [19]). (2.12) implies that in both the Petrov $\mathrm{N}$ and conformally flat cases the terms $\sigma \Psi_{4}$ and $\kappa \Psi_{4}$ which would otherwise appear in $(\mathrm{Bg})$ and $(\mathrm{Bh})$ respectively are zero.

For $s_{1}=2$ one also has

$$
\alpha=\beta+\tau=0,
$$


while for $s_{1}=1$ (with, as usual, $B$ imaginary) one has

$$
\alpha=\beta+\tau \text {. }
$$

For $\Psi,\left(\mathrm{A}_{2}\right)$ requires that $\nabla^{2} \Psi$ satisfies

$$
\begin{aligned}
\text { (i) } \nabla^{2} \Psi_{A B^{\prime}} & =0 \text { for } A+B=0, \ldots 5 \\
\text { (ii) } 6 \nabla^{2} \Psi_{51^{\prime}} & =\nabla^{2} \Psi_{60^{\prime}} \\
\text { (iii) } 5 \nabla^{2} \Psi_{42^{\prime}} & =2 \nabla^{2} \Psi_{51^{\prime}} \\
\text { (iv) } 3 \nabla^{2} \Psi_{52^{\prime}} & =\nabla^{2} \Psi_{61^{\prime}},
\end{aligned}
$$

In general the conditions on higher derivatives to ensure that $\left(\mathrm{A}_{m}\right), m>2$, is satisfied do not give new conditions on the curvature and spin coefficients, so the relevant conditions, which were used in checking invariances, will not be written out here.

\subsection{The Bianchi identities}

The Bianchi identities with the canonical choices of frame given in Section 2.1 are written out in Appendix B. Some immediate consequences of those Bianchi identities are collected here.

When all $\Phi_{A B^{\prime}}=0,(\mathrm{Bh})$ and $(\mathrm{Bk})$ give $\delta \Lambda=0=\Delta \Lambda$ and for LNRI $D \Lambda=0$ so $\Lambda$ is a constant.

In Segre type $[(1,3)](\mathrm{Bb})$ shows $\kappa=0,(\mathrm{Bh})$ reads $\delta \Lambda=\rho \Phi_{12^{\prime}}$ which shows that $\rho$ is real and $(\mathrm{Bg})$ then implies $\varepsilon=0$. Compatibility with $(\mathrm{Bj})$ then implies $\sigma=0$. (Bf) shows that $\beta$ is real. Eliminating $\delta \Phi_{12^{\prime}}$ and $\Delta \Lambda$ between $(\mathrm{Bc}),(\mathrm{Bf})$ and $(\mathrm{Bk})$ then implies

$$
\alpha+\tau+(\pi+\bar{\pi})=\beta
$$

Substituting back into (Bf) then shows that $\alpha$, and therefore $\tau$, are real.

In Segre type [1(1,2)], (Be) gives $\rho+\bar{\sigma}=0,(\mathrm{Bb})$ shows $\varepsilon$ is real, $(\mathrm{Bh})$ shows $\alpha-\bar{\beta}$ is real and $(\mathrm{Bj})$ then implies that $\kappa$ is real. However $(\mathrm{Ba})$ gives $\kappa+\bar{\kappa}=0$, so $\kappa=0$ and then $(\mathrm{Bg})$ and $(\mathrm{Bh})$ give (2.9). Elimination between $(\mathrm{Bh})$ and $(\mathrm{Bj})$ gives (2.8) so $\lambda+\bar{\mu}$ is a null rotation invariant, and

$$
(\alpha+\bar{\alpha})=(\beta+\bar{\beta})+(\tau+\bar{\tau})
$$

Hence one can write $\alpha$ in terms of $\beta$ and $\tau$.

From [11], in Segre type [(11,2)] $\delta \Lambda=0=\kappa$, and $\Delta \Lambda=\rho=-2(\varepsilon+\bar{\varepsilon})$. In Petrov type $\mathrm{N}, \rho=\epsilon=0=\sigma$ follows from (2.12), so $\Lambda$ is constant, while in the conformally flat case (Bc) implies $\sigma=0$ and, setting $\Phi_{22^{\prime}}=1, \bar{\tau}=2(\alpha-\bar{\beta})$ from $(\mathrm{Bd})$.

In Segre type [1(11,1)], $(\mathrm{Be})$ gives $\rho+\bar{\sigma}=0,(\mathrm{Bb})$ implies that $\varepsilon$ is real and $(\mathrm{Ba})$ that $\kappa$ is imaginary. Then the real parts of $\tau$ and $\pi$ are null rotation invariants, (Bf) 
shows that $\mu+\bar{\lambda}$ is real, and $(\mathrm{Bg})$ and (Bh) show that $\alpha-\bar{\beta}$ is real and (2.9) holds. (Bj) and (Bh) imply $\delta\left(\Phi_{11^{\prime}}-\Lambda\right)=-2(\pi+\bar{\pi}) \Phi_{11^{\prime}}$ and (2.8). From (2.8), $\alpha$ can be written in terms of $\beta$ and $\tau$ (or $\pi$ ). (Bf) and (Bk) yield $\Delta\left(\Phi_{11^{\prime}}-\Lambda\right)=0$.

\subsection{LNRI and derivatives of $\Phi$}

For $\left(\mathrm{A}_{1}\right)$ the non-zero Ricci curvature components satisfy $D Q=0$ and $\delta Q=\bar{\delta} Q$ as discussed in Sect. 2.1. When there is a two-dimensional LNRI group $\left(\mathrm{A}_{1}\right)$ further requires that $\nabla \Phi_{33^{\prime}}$ and $\nabla^{2} \Phi_{44^{\prime}}$ are the only non-zero components of $\nabla \Phi_{A B^{\prime}}$ and $\nabla^{2} \Phi_{A B^{\prime}}$.

When there is a one-parameter LNRI group (with imaginary $B$ ), applying (2.2) and (2.3) shows $\left(\mathrm{A}_{1}\right)$ implies the following requirements on $\nabla \Phi_{A B^{\prime}}$ :

$$
\begin{aligned}
\text { (i) } \nabla \Phi_{A B^{\prime}} & =0 \text { for } A+B=0, \ldots 2 \\
\text { (ii) } 3 \nabla \Phi_{21^{\prime}} & =\nabla \Phi_{30^{\prime}} \\
\text { (iii) } 3 \nabla \Phi_{22^{\prime}} & =2 \nabla \Phi_{31^{\prime}} \\
\text { (iv) } \nabla \Phi_{32^{\prime}} & \text { is real. }
\end{aligned}
$$

To complete the study of $\left(\mathrm{A}_{1}\right)$ one needs to consider not only $\nabla \Psi$ and $\nabla \Phi$ but also $\Xi_{A B^{\prime}}$ (as defined by $\Xi_{C D E W^{\prime}}=\nabla^{C}{ }_{W^{\prime}} \Psi_{C D E F}$, [13]), which is zero in the conformally flat case. The conditions on $\Xi_{A B^{\prime}}$ arising from (2.2)-(2.3) are usually trivial to check.

With LNRI of $\Phi, \nabla \Phi_{00^{\prime}} \equiv 0$, (Ba) implies $\nabla \Phi_{01^{\prime}}=0$ and (Bb) implies $\nabla \Phi_{02^{\prime}}=0$, so (i) above is satisfied. The remaining conditions are best inspected case by case (see below).

The tests to be applied to $\nabla^{2} \Phi$ for $\left(\mathrm{A}_{2}\right)$ are as follows.

$$
\begin{aligned}
\text { (i) } \nabla^{2} \Phi_{A B^{\prime}} & =0 \text { for } A+B=0, \ldots 3 \\
\text { (ii ) } 4 \nabla^{2} \Phi_{31^{\prime}} & =\nabla^{2} \Phi_{40^{\prime}} \\
\text { (iii ) } 3 \nabla^{2} \Phi_{22^{\prime}} & =2 \nabla^{2} \Phi_{31^{\prime}} \\
\text { (iv) } 4 \nabla^{2} \Phi_{32^{\prime}} & =2 \nabla^{2} \Phi_{41^{\prime}} \\
\text { (v) } 4 \nabla^{2} \Phi_{33^{\prime}} & =3 \nabla^{2} \Phi_{42^{\prime}} \\
\text { (vi }) \nabla^{2} \Phi_{43^{\prime}} & \text { is real. }
\end{aligned}
$$

For $\left(\mathrm{A}_{m}\right), m>1$, one has to consider, as well as $\nabla^{m}$ applied to $\Psi_{A}, \Phi_{A B^{\prime}}$ and $\Lambda$, the derivatives of order $(m-1)$ of $\Xi_{A B^{\prime}}$ in Petrov type $\mathrm{N}$, and higher derivatives of terms computed at previous levels of differentiation (see [13]). The required conditions on these for LNRI are readily inferred from (2.2)-(2.3).

\section{LNRI spacetimes}

The minimal $m$ in $\left(\mathrm{A}_{m}\right)$ with LNRI that would ensure an isometry group as in the criterion (C) quoted in Section 1 will now be studied. In the Petrov type D cases with spatial rotation or boost invariance treated in the previous papers [11,12], all allowable 
Ricci tensors could be treated together. For LNRI spacetimes of Petrov type $\mathrm{N}$ this cannot readily be done and treating the possible Ricci tensor types separately seems necessary. That also allows one to treat the conformally flat and Petrov $\mathrm{N}$ cases with the same Ricci tensor type together.

The strategy in doing so is similar to that used in the previous papers of this series. One first fixes the frame so that the curvature has an appropriate canonical form, as set out in section 2.1. Then one can derive the implications of $\left(\mathrm{A}_{1}\right)$ together with the Bianchi identities, as set out in Sections 2.2-2.4. The Bianchi identities, as given in Appendix B, are used together with the canonically nonzero components of curvature to extract conditions on the spin coefficients, and the implications of $\left(A_{1}\right)$ such as (2.18) are used to find further conditions. The commutator relations, NP equations and $\left(\mathrm{A}_{m}\right), m \geq 2$, then remain to be considered.

The literature on known metrics for LNRI spacetimes, mostly of Petrov type N, surveyed in Appendix A, covers the main classes of spacetime which can be obtained by assuming $\left(\mathrm{A}_{m}\right)$ with LNRI for sufficiently large $m$. However, more detailed consideration is needed to find the minimal $m$ for each case that would ensure an isometry group as in condition (C) in Section 1 .

\subsection{LNRI spacetimes with $\Phi_{A B^{\prime}}=0$}

These are either the well-known conformally flat spacetimes of constant curvature, which need not be considered further, or are Petrov type $\mathrm{N}$ Einstein spaces. For these spacetimes it will be shown that $\left(\mathrm{A}_{2}\right)$ is sufficient to prove local null rotation isotropy in all cases, but $\left(\mathrm{A}_{1}\right)$ suffices if the LNRI group is two-dimensional.

In a canonically chosen frame $\Psi_{4}$ is constant and the Bianchi identities imply $\Lambda$ is constant. So $t_{0}=0$ and, from (2.11), the only first derivatives of the curvature that could be nonzero are $\nabla \Psi_{41^{\prime}}, \nabla \Psi_{50^{\prime}}$ and $\nabla \Psi_{51^{\prime}}$. (Bc) gives $4 \varepsilon-\rho=0$, so $\varepsilon=\rho=0$, using (2.12), and (Bd) gives $4 \beta-\tau=0$.

If $s_{1}=2$, these and (2.13) imply

$$
\alpha=\beta=\varepsilon=\kappa=\rho=\sigma=\tau=0 .
$$

In this case $k_{a ; b}=-(\gamma+\bar{\gamma}) k_{a} k_{b}$, so $\boldsymbol{k}$ is proportional to (but not necessarily equal to) a covariantly constant null vector ${ }^{4}$. Thus the spacetimes are $p p$-waves, (A.1) (see [19], Section 24.5).

With (3.1), (NPl) shows that $\Lambda=0$. Inspection of (2.11) shows that the only possible functions of position in the first derivatives are the real and imaginary parts of $\gamma$. The only component among the minimal set of second derivative terms defined in [13] which is not identically zero is $\nabla^{2} \Psi_{62^{\prime}}=\left(4 \Delta \gamma+4 \gamma \bar{\gamma}+20 \gamma^{2}\right) \Psi_{4}$, and similarly at the third derivative only $\nabla^{3} \Psi_{73^{\prime}}$, which is a combination of $\gamma$, its complex conjugate and $\Delta$ derivatives thereof, can be nonzero. So $\left(\mathrm{A}_{1}\right)$ implies $\left(\mathrm{A}_{3}\right)$, whence $t_{1} \leq 1$ so there can be at most one independent function in $\gamma$, say $w$. If $w$ is constant the CartanKarlhede procedure terminates at the first step with $s_{1}=2, t_{1}=0$ and there is a

\footnotetext{
${ }^{4}$ In general $k_{a ; b}$ is $(\alpha+\bar{\beta}) k_{a} m_{b}+\tau \bar{m}_{a} k_{b}-\rho \bar{m}_{a} m_{b}-\sigma \bar{m}_{a} \bar{m}_{b}+\kappa \bar{m}_{a} l_{b}+(C C)-(\gamma+\bar{\gamma}) k_{a} k_{b}-(\varepsilon+\bar{\varepsilon}) k_{a} l_{b}$, where $(C C)$ stands for the complex conjugate of all the preceding terms.
} 
group $G_{6}$. These are (locally) the well-known homogeneous plane wave spacetimes ((12.12) in [19]). Otherwise $t_{1}=1=t_{2}, s_{2}=2$ and the Cartan-Karlhede procedure terminates at step 2. These are (locally) the $p p$-waves in which there is a $G_{5}$ transitive on three-dimensional null surfaces ((24.46) in [19]).

Thus Petrov type $\mathrm{N}$ spacetimes with $\Phi_{A B^{\prime}}=0$ admitting a two-dimensional LNRI group are $p p$-waves (including plane waves), and $\left(\mathrm{A}_{1}\right)$ is a sufficient criterion.

Now consider $s=1$ (with imaginary parameter $B$ ). From $(\mathrm{Bc}),(\mathrm{Bd})$ and the results of Sect. 2.2

$$
\kappa=\sigma=\rho=\epsilon=0, \quad \tau=4 \beta, \quad \alpha=5 \beta .
$$

If $\beta=0$ the spacetime will have $s=2$ so $\beta \neq 0$ if $s=1$. (NPp), (NPq) and (NPl) read respectively

$$
\begin{aligned}
4 \delta \beta & =20 \beta^{2}-20 \beta \bar{\beta}, \\
4 \bar{\delta} \beta & =20 \beta^{2}+12 \beta \bar{\beta}+2 \Lambda, \\
5 \delta \beta-\bar{\delta} \beta & =26 \beta \bar{\beta}-10 \beta^{2}+\Lambda,
\end{aligned}
$$

which imply $\Lambda=20 \beta^{2}-36 \beta \bar{\beta}$. Thus $\beta^{2}$ must be real, so $\beta$ is either real or pure imaginary, and in either case since $\Lambda$ is constant $\beta$ is constant. Thus the only possible independent function(s) of position in the first derivatives of the curvature tensor are in $\gamma$. With (3.2), $\gamma$ is an invariant so $D \gamma=0$. Then (NPf) implies $\beta(\pi+\bar{\pi})=-8(\beta \bar{\beta})$, which implies $\beta$ is real, and $(\pi+\bar{\pi})=-8 \beta$. Then (2.8) and (2.9) are true and $\lambda+\bar{\mu}$ is an invariant.

(NPo) and (NPr) give

$$
\begin{aligned}
& \delta \gamma=\beta(-3 \gamma+\bar{\gamma}+5(\mu+\bar{\lambda})), \\
& \bar{\delta} \gamma=\beta(3 \gamma-5 \bar{\gamma}+5(\lambda+\bar{\mu})) .
\end{aligned}
$$

Since $\gamma$ is an invariant, $\delta \gamma=\bar{\delta} \gamma$ so $6 \gamma+5(\lambda+\bar{\mu})$ is real. With this and earlier information, $\left(\mathrm{A}_{2}\right)$ can be checked. Computing $\nabla^{2} \Psi$ one finds (2.15) (i)-(iii) are satisfied, but (2.15) (iv) requires $\gamma$ to be real, so $(\mu+\bar{\lambda})$ is also real, and (2.10) is satisfied whence $v+\bar{v}$ is an invariant. $\nabla^{2} \Psi_{62^{\prime}}=\left[-20(v+\bar{v})+24 \gamma^{2}+\Delta \gamma\right] \Psi_{4}$, whence $v+\bar{v}$ could be a further independent function of position.

Continuing to the further derivatives, using the above information, shows $\left(\mathrm{A}_{3}\right)$ and $\left(\mathrm{A}_{4}\right)$ are satisfied. Thus $\left(\mathrm{A}_{2}\right)$ ensures a one-dimensional null rotation isotropy group in Petrov type $\mathrm{N}$ if $\Phi_{A B^{\prime}}=0$, but unless a different way to prove $\gamma$ is real has been overlooked, $\left(\mathrm{A}_{1}\right)$ is insufficient. The spacetime has a $G_{3}$ if $t_{p}=2$, or possibly a $G_{r}$, $r>3$ and is either in the (A.2) or (A.8) classes. As set out in Appendix A, there are known LNRI Petrov N Einstein spaces in these classes, including the Kaigorodov metric with a $G_{5}$. 


\subsection{LNRI spacetimes with a Ricci tensor of Segre type $[(1,3)]$}

Here it is fairly straightforward to prove that $\left(A_{1}\right)$ is sufficient to ensure local null rotation isotropy.

Using the implications found in Sect. 2.3 from the Bianchi identities, and applying $\left(\mathrm{A}_{1}\right)$ to $\nabla \Phi$ one finds that (2.18) (i) is true, (ii) implies $\rho=0$, and (iii) together with (2.16) implies (2.9), (2.8) and $\alpha=\beta+\tau$. (2.18) (iv) implies that $2 \gamma+\bar{\lambda}+\mu$ is real, i.e. (2.10) is true. In the conformally flat case (Bd) implies $\gamma+\lambda+\bar{\mu}$ is real so $\gamma$ and $\lambda+\bar{\mu}$ are both real.

Hence, whether $\Psi_{4} \neq 0$ or not, (2.7) holds if $\left(\mathrm{A}_{1}\right)$ is true; $\alpha, \beta$ and $\tau$ are real; and $\alpha, \beta, \gamma, \tau, \lambda+\bar{\mu}$ and $\nu+\bar{v}$ are invariants. (Bc) now gives $\delta \Phi_{12^{\prime}}=-2 \beta \Phi_{12^{\prime}}$ and (Bf) that $\Delta \Lambda=(\pi+\bar{\pi}) \Phi_{12^{\prime}}$. Note that in the conformally flat case there is still the freedom to boost to a frame such that $\left|\Phi_{12^{\prime}}\right|=1$.

(NPp) gives $\delta \tau=0$ and then (NPf), (NPl) and (NPq) each yield $\Lambda=-\tau^{2}$. If $\tau=0$ then (2.9) implies $\pi+\bar{\pi}=0$. Otherwise $-2 \tau \Delta \tau=\Delta \Lambda=(\pi+\bar{\pi}) \Phi_{12^{\prime}}=-2 \tau \Phi_{12^{\prime}}$ implies $\Delta \tau=\Phi_{12^{\prime}} \neq 0, \tau$ is a non-constant invariant, and $t_{p} \geq 1$.

$\left(\mathrm{A}_{2}\right)$ and $\left(\mathrm{A}_{3}\right)$ are now identically fulfilled in all cases above as a result of the constraints found, regardless of the values of $\Psi_{4}$ and $\tau$. Since $s_{i}=1$ for all $i$ the spacetime must have at least a $G_{3}$. Of the metrics in Appendix A only (A.2), with a $G_{3}$ on $N_{3}$, can support a Ricci tensor of Segre type [(1,3)], and an example with this Segre type is given there.

\subsection{LNRI spacetimes with a Ricci tensor of Segre type $[1(1,2)]$}

As in the case of Segre type [(1,3)], $\left(\mathrm{A}_{1}\right)$ is sufficient to ensure local null rotation isotropy, but the calculations are a little more complicated in the conformally flat case, so that case is treated first.

Using the results already stated in Section 2.3. (Bd) implies that $\bar{\tau}-2 \bar{\beta}-2 \alpha$ is real so using the reality of $\alpha-\bar{\beta}, \tau-\bar{\tau}=4(\beta-\bar{\beta})$. Adding the imaginary parts of (Bc) and (Bf) gives (2.10). On substituting for $\Delta \Phi_{11^{\prime}}$ and $\Delta \Lambda$ from the real parts of (Bc) and (Bf) into (Bk) one obtains $\bar{\rho}+\rho+4 \varepsilon=0$.

Now let $\left(\mathrm{A}_{1}\right)$ be imposed. (2.18) (i) and (ii) are identically satisfied. Evaluating the imaginary part of (2.18) (iii), using (2.10), implies that $\gamma$ and $\bar{\lambda}+\mu$ are real. Now (Bf) implies $\rho$ is real. The real part of (2.18) (iii) gives $2 \rho+\varepsilon=0$ so $\rho=\varepsilon=0$, and therefore $\sigma=0$. Evaluating (2.18) (iv) then shows that $\beta$ is real and hence $\alpha$ and $\tau$ are also real and (2.17) becomes $\alpha=\beta+\tau$. So (2.7) holds. With these conditions $\left(\mathrm{A}_{2}\right)$ and $\left(\mathrm{A}_{3}\right)$ hold and the spacetime has a group $G_{3}, r \geq 3$.

From (NPp) and $(\mathrm{NPq}), \delta \tau=2 \Phi_{11^{\prime}}=2\left(\tau^{2}+\Lambda\right)$ so $\tau$ cannot be constant (and hence $\left.t_{p} \geq 1\right)$. Since $\delta\left(\Phi_{11^{\prime}}-\Lambda\right)=4 \tau \Phi_{11^{\prime}} \neq 0, t_{0} \geq 1$. Thus $t_{1}$ could be 1 ; then the Cartan-Karlhede procedure terminates at the first step and there is a $G_{4}$, which might allow a metric of the form (A.10), while if $t_{p}=2$, the Cartan-Karlhede procedure stops at step 2 or 3, giving a Barnes metric, (A.2). One can also infer from (NPo) and (NPr) that $\Delta \tau=\Delta(\alpha-\beta)=0$.

In the Petrov N case, since $\sigma=0=\rho+\varepsilon$ from (2.12), (Be) implies $\rho=\varepsilon=0$. (2.18) will give the same equations as when $\Psi_{4}=0$, so again $\alpha, \beta$ and $\tau$ are all real 
and $\alpha=\beta+\tau, \gamma$ is real and so is $\bar{\lambda}+\mu$, and all these are invariants. These conditions are useful in checking $\left(\mathrm{A}_{2}\right)$, which is now easily found (with CLASSI's help) to be satisfied, as is $\left(\mathrm{A}_{3}\right)$. The deductions in the previous paragraph apply to the Petrov type $\mathrm{N}$ case also. An example is given in Appendix A.

\subsection{Petrov N LNRI spacetimes with a Ricci tensor of Segre type $[(11,2)]$}

For these spacetimes it will be shown that in some cases, namely those with $s=1$ and $\alpha=0=\beta+\tau$ discussed below, $\left(\mathrm{A}_{2}\right)$ is required to ensure local null rotation isotropy. Otherwise $\left(\mathrm{A}_{1}\right)$ suffices.

Although this Segre type of Ricci tensor is invariant under a three-dimensional group, these spacetimes have only a two-dimensional LNRI group at the initial step of the Cartan-Karlhede procedure, i.e. $s_{0}=2$, since the spatial rotation invariance of $\Phi_{A B^{\prime}}$ cannot be shared by the Weyl tensor. The calculations are somewhat similar to those for the Petrov type $N$ Einstein spaces above. With the usual choice of frame, (2.12) and (2.14) hold and if $s_{1}=2$ so does (2.13). (Bc) then implies $\varepsilon=\rho=0$, so if $s=1,(2.7)$ is true. Note that $\alpha, \beta, \gamma, \tau$, and $\pi+\bar{\pi}$ are invariants whether $s=1$ or 2 .

When $s=2$, so $\delta \Phi_{22^{\prime}}=0$, then (2.13) and the Bianchi identity not yet used, (Bd), give

$$
5 \beta \Psi_{4}=3 \bar{\beta} \Phi_{22^{\prime}}
$$

If $s=\mathbf{2}$ and $\boldsymbol{\beta} \neq \mathbf{0}$, taking moduli in (3.4) yields that $\Phi_{22^{\prime}}= \pm 5 / 3$. (NPl) and (NPq) give $\Lambda=-\beta \bar{\beta} \leq 0$. Since, as in Sect. $2.3 \Lambda$ is constant, $|\beta|$ is constant and $t_{0}=0$. A constant spatial rotation could be used to make $\beta$ real. Since $\gamma$ is a null rotation invariant, $\delta \gamma=0$ and (NPo) implies $\gamma=0$. Inspection shows that $t_{1}=0$ and the Cartan-Karlhede procedure terminates at the first step. The resulting spacetime is the solution of Defrise [2] admitting a $G_{6}$, which appears as (12.6) in [19]. (In [19] this metric was arrived at by assuming the $G_{6}$ from the start, but the arguments are closely analogous.)

If $\boldsymbol{s}=\mathbf{2}$ and $\boldsymbol{\beta}=\mathbf{0},(3.1)$ holds and as in Sect. 3.1 the spacetimes are $p p$-waves. Following the arguments in that Section, $\Lambda=0$, and as $\gamma$ is a null rotation invariant, its only possible nonzero derivative is $\Delta \gamma$. Unless $t_{0}=0$ and $t_{1}=1$ the Cartan-Karlhede procedure terminates at step 1 , and otherwise at step 2 , with respectively a $G_{5}$ if $t_{1}=1$ or a $G_{6}$ if $t_{1}=0$. The possible metrics have been discussed in [18] (see Appendix A). For these cases only $\left(\mathrm{A}_{1}\right)$ was needed.

If $s=\mathbf{1},(\mathrm{NPp})$ and (NPq) imply $\Lambda=-\tau \bar{\tau}$ so $|\tau|$ is constant, as in the case $s=2$ with $\beta \neq 0=\beta+\tau$. Substituting into (NPl), remembering that $\beta$ is a null rotation invariant, shows that $\alpha(2(\alpha-\bar{\alpha})-(\tau-\bar{\tau}))=0$. Hence two cases result, depending on whether $\alpha=0=\beta+\tau$ or not.

If $s=\mathbf{1}$ and $\boldsymbol{\alpha}=\mathbf{0}=\boldsymbol{\beta}+\boldsymbol{\tau}$, then $\delta \Phi_{22^{\prime}}=0$ would imply $s_{1}=2$ by inspection of the only non-zero quantities with $q=1$ in the minimal set of [13]. One also has (3.4), and by the same arguments as above one arrives at the Defrise metric, (A.9), or $p p$-waves. So one must have $\delta \Phi_{22^{\prime}} \neq 0$, whence $t_{0}=1$ ( $\Psi_{4}$ and $\Lambda$ being constant), and $\left(\mathrm{A}_{4}\right)$ will not be required. To consider $\left(\mathrm{A}_{2}\right)$, note that from $(\mathrm{NPp}), \delta \tau=0=\delta \beta$, 
whence (2.15) is satisfied. From (2.19) (v) with $\delta \Phi_{22^{\prime}} \neq 0,2 \bar{\beta}=\pi+\bar{\pi}$ so $\beta$ and $\tau$ are real and constant. (2.9) and (2.8) are true. If $\beta \neq 0 \neq \Lambda$, (NPo) and (NPr) imply $\gamma$ is real. (2.19) (vi) implies (after use of the $[\delta, \Delta]$ commutator) that $\lambda+\bar{\mu}$ is also real. Then $\left(\mathrm{A}_{3}\right)$ is true and there is at least a $G_{3}$, assuming $\left(\mathrm{A}_{2}\right)$.

If $s=\mathbf{1}$ and $\boldsymbol{\alpha} \neq \mathbf{0}$, then $(\tau-\bar{\tau})=-2(\beta-\bar{\beta})$ and $(\alpha-\bar{\alpha})=(\tau-\bar{\tau})+(\beta-\bar{\beta})=$ $-(\beta-\bar{\beta}) .\left(\mathrm{A}_{1}\right)$ is then satisfied. Since $\gamma$ is an invariant, (NPf) implies (2.9) (and thus (2.8)). Checking $\left(\mathrm{A}_{2}\right),(2.15)$ (ii) and (iii) are then satisfied. (2.15) (iv) gives (2.10). Conditions (2.19) are then satisfied. The quantities $\gamma, \lambda+\bar{\mu}$ and $v+\bar{v}$ are invariants. Using this information, $\left(\mathrm{A}_{3}\right)$ is then satisfied. The curvature components other than $\Phi_{22^{\prime}}$ are constants, so $t_{0} \leq 1$ while $s_{0}=2$ and $s_{1}=1$. If $\Phi_{22^{\prime}}$ is not constant, then since $t_{2} \leq 2$, the Cartan-Karlhede procedure will terminate at the latest at $p=2$ (with an $(s, t)$ sequence $(2,1) \rightarrow(1,1) \rightarrow(1,2)$ ) and there will be a $G_{3}$ or, if $t_{2}=t_{1}=1$, a $G_{4}$. Only if $\Phi_{22^{\prime}}$ is constant and $t_{0}=t_{1}=0$ is a check of $\left(\mathrm{A}_{4}\right)$ required. The inferences above from $\left(\mathrm{A}_{1}\right)$ and $\left(\mathrm{A}_{2}\right)$ remain true and $t_{1}=0$ implies that (from (2.11)) that $\alpha$ and $\gamma$ are constants as well as $\Phi_{22^{\prime}}$. With these extra conditions $\left(\mathrm{A}_{4}\right)$ is readily checked (using CLASSI).

\subsection{Conformally flat LNRI spacetimes with a Ricci tensor of Segre type $[(11,2)]$}

As for the Petrov type $\mathrm{N}$ spacetimes with Ricci tensor of Segre type [(11,2)] just considered, some subcases (those with $\tau=0$ below) require $\left(\mathrm{A}_{2}\right)$ to ensure local null rotation isotropy, while otherwise $\left(\mathrm{A}_{1}\right)$ suffices.

By Theorem 37.19 of [19], all conformally flat pure radiation spacetimes (with $\Lambda=0)$ are included in the metrics (37.104)-(37.106) there. Here the $m$ required in $\left(\mathrm{A}_{m}\right)$ to ensure that an LNRI spacetime (allowing $\Lambda \neq 0$ ) has null rotation isotropy is considered. Direct calculation shows that imposing null rotation invariance on (37.106) of [19] leads (from (2.19) (v)) to $\Phi_{22^{\prime}}=0$, i.e. this case will not arise. With a zero Weyl tensor there is still full boost and spatial rotation freedom: the boost can and will be used to set $\Phi_{22^{\prime}}= \pm 1$, and if $s=1$ the rotation will be used to make $B$ imaginary.

Summarizing earlier arguments, $(\mathrm{Bc})$ gives $\sigma=0,(\mathrm{Bg}, \mathrm{h}$ and i) give $\kappa=0=\delta \Lambda$, (Bf) and (Bk) imply that $\rho$ is real, $\Delta \Lambda=\rho \Phi_{22^{\prime}}$ and $\rho=-2(\varepsilon+\bar{\varepsilon})$. (2.18) (iii) then implies $\varepsilon+\bar{\varepsilon}=0=\rho$ (so $\varepsilon$ is imaginary and $\tau$ is an invariant), and $\Lambda$ is constant. Thus $t_{0}=0$. (Bd) implies that $\bar{\tau}=2 \bar{\beta}+2 \alpha$, while (2.18) (iv) requires $\tau+\bar{\alpha}+\beta$ to be real. Thus $\tau=2(\alpha+\bar{\beta})$ is real. The imaginary part of (NPp) now implies $\tau=0$ or $\beta-\bar{\alpha}$ is real, so $\alpha$ and $\beta$ are both real.

If $\boldsymbol{\tau}=\boldsymbol{\alpha}+\overline{\boldsymbol{\beta}}=\mathbf{0}, \boldsymbol{k}$ is proportional to a covariantly constant vector, as in Sect. 3.1, and the spacetime is a $p p$-wave with a $G_{5}$. These cases have a 2-dimensional LNRI group and are LRS so $s_{1}=3$. They are the solutions (37.104)-(37.105) in [19].

If $\tau \neq \mathbf{0}, \alpha, \beta$ and $\tau$ are all real and $\alpha+\beta \neq 0$. Then (NPq) gives $\Lambda=-2\left(\alpha^{2}-\beta^{2}\right)$. The imaginary part of (NPc) implies $\varepsilon-\bar{\varepsilon}=0$ so $\varepsilon$ is zero and $\alpha$ and $\beta$ are invariants. Then (2.18) is satisfied. Since $\nabla \Phi_{23^{\prime}}=\tau \Phi_{22^{\prime}}= \pm \tau \neq 0, s_{1}=1=s$.

Checking ( $\mathrm{A}_{2}$ ), (2.19) (i)-(iv) are automatically satisfied, while (2.19) (v) gives $\alpha+3 \beta=0$ so $\alpha=-3 \beta, \tau=-4 \beta$ and $\Lambda=-\tau^{2} \neq 0$. Thus $\tau, \alpha$ and $\beta$ are constants. (2.14) holds so $\gamma$ is an invariant. (NPf) now gives (2.9) or equivalently (in this case) (2.8), and (2.19) (vi) gives (2.10). So $\lambda+\bar{\mu}$ and $v+\bar{v}$ are invariants. Using 
(2.10), (NPo) and (NPr) now imply $\gamma$ and $\lambda+\bar{\mu}$ are real, and $\delta \gamma=-2 \beta \gamma-3 \beta(\lambda+\bar{\mu})$. $\left(\mathrm{A}_{3}\right)$ is then satisfied.

Since $s_{0}=3$ and $s_{1}=1$, conditions arising from $\left(\mathrm{A}_{4}\right)$ might be required to prove null rotation isotropy if $t_{1}$ were zero. This would require $\gamma$ to be constant and $\lambda+\bar{\mu}$ to be zero. With these conditions, $\left(\mathrm{A}_{4}\right)$ is readily checked (using CLASSI). If $\gamma$ is not constant, $t_{1}=1$. Among the second derivatives, only $\nabla^{2} \Phi_{44^{\prime}}=12(v+\bar{v})+24 \gamma^{2}+\Delta \gamma$ could contain another function of position. If it does, the Cartan-Karlhede procedure terminates with $p=2$ and $t_{p}=2$ and otherwise with $p=1$ and $t_{p}=1$.

\subsection{LNRI spacetimes with a Ricci tensor of Segre type $[1(11,1)]$}

For this Segre type, $\left(A_{1}\right)$ suffices to ensure local null rotation isotropy in the Petrov type $\mathrm{N}$ cases, but $\left(\mathrm{A}_{2}\right)$ is required for some conformally flat cases.

In the Petrov type $\mathrm{N}$ case, the relations given in Section 2.3, and (2.12) and (2.14), which follow from $\left(\mathrm{A}_{1}\right)$, together give (2.7). Hence whatever the value of $\Psi_{4}, \varepsilon \Psi_{4}=0$ and $(\mathrm{Bc})$ then shows $\gamma$ is real. $(\mathrm{Bg}),(\mathrm{Bh})$ and $(\mathrm{Bj})$ show $\alpha-\bar{\beta}$ is real and (2.9) and (2.8) are true. (Bf) implies $\lambda+\bar{\mu}$ is real. Thus (2.10) is true and $\lambda+\bar{\mu}$ and $v+\bar{v}$ are invariants. Reality of $\alpha-\bar{\beta}$ and (2.14) give $\tau-\bar{\tau}=-2(\beta-\bar{\beta})=2(\alpha-\bar{\alpha})$. ( $\left.\mathrm{A}_{1}\right)$, $\left(\mathrm{A}_{2}\right)$ and $\left(\mathrm{A}_{3}\right)$ are then satisfied. As $s_{0}=3$ while $s_{1}=1$, one must check if $\left(\mathrm{A}_{4}\right)$ is needed to ensure null rotation isotropy. However, the conditions for $t_{0}=t_{1}=0$ are very strong $\left(\Lambda, \Phi_{11^{\prime}}, \alpha, \gamma, v+\bar{v}, \tau+\bar{\tau}\right.$ and $\beta+\bar{\beta}$ must be constants and $\left.\lambda+\bar{\mu}=0\right)$, and with these conditions $\left(\mathrm{A}_{4}\right)$ is satisfied. The spacetime has at least a $G_{3}$.

In the conformally flat case, $\left(\mathrm{A}_{1}\right)$ is again satisfied, as a consequence of the Bianchi identities, and from (Bd) $v+\bar{v}=0$. (2.19) (i) and (vi) are then satisfied.

If $(\boldsymbol{\mu}+\bar{\lambda}) \neq \mathbf{0}$, then (2.19) (ii) and (iii) imply that $\rho$ is real and $\varepsilon+2 \rho=0$, while (2.19) (iv) implies that $2 \beta+\tau$ is real. As $\alpha-\bar{\beta}$ is invariant, the imaginary part of (NPd) added to the conjugate of (NPe) yields that $\kappa=0$. Then $\rho$ is a null rotation invariant, so $D \rho=0$ in $(\mathrm{NPa})$ which implies $\rho=\sigma=\varepsilon=0$. Then $\left(\mathrm{A}_{3}\right)$ is satisfied, and since $t_{0} \geq 1$ the Cartan-Karlhede procedure must terminate at $p=1$ or $p=2$. $\left(\mathrm{A}_{2}\right)$ implies null rotation isotropy in this case.

If $(\boldsymbol{\mu}+\bar{\lambda})=\mathbf{0}$, no information arises from $\left(\mathrm{A}_{2}\right)$. In this case, from (Bc, $\mathrm{f}$ and $\left.\mathrm{k}\right)$ $\Delta \Lambda=\Delta \Phi_{11^{\prime}}=0$. The only spin coefficient combination appearing in the symmetrized spinor derivatives of $\Phi$ is $\tau+\bar{\tau}$ and its derivatives and $\delta\left(\Phi_{11^{\prime}}-\Lambda\right)=$ $(\tau+\bar{\tau}) \Phi_{11^{\prime}}($ from $(\mathrm{Bg}, \mathrm{h}$ and $\mathrm{j}))$. Applying the $[\delta, \Delta]$ commutator to $\left(\Phi_{11^{\prime}}-\Lambda\right)$ gives $\Delta(\tau+\bar{\tau})=0 .\left(\mathrm{A}_{2}\right)$ and $\left(\mathrm{A}_{3}\right)$ are then satisfied. If $t_{0}=t_{1}=0$, so $\Phi_{11^{\prime}}, \Lambda$ and $\tau+\bar{\tau}$ are constant, $\left(\mathrm{A}_{4}\right)$ could be required to ensure the Cartan-Karlhede procedure terminates at $p \leq 3$, but inspection shows that then $t_{2}=0$ also, so the Cartan-Karlhede procedure will terminate with $p=1$. In this subcase only $\left(\mathrm{A}_{1}\right)$ has been used, and only for directional derivatives of $\Phi_{11^{\prime}}$ and $\Lambda$.

\section{Conclusion}

In this paper, it has been shown that the conjecture of Siklos, although not correct in general, as discussed in the introduction to Paper 1 of this series [11], is correct in 
almost all cases with local null rotation invariance. Thus, as stated in the introduction, the following holds:

Theorem 1 Einstein spacetimes with a Weyl tensor of Petrov type N, spacetimes with a Ricci tensor of Segre type $[(11,2)]$, and conformally flat spacetimes with a Ricci tensor of Segre type $[1(11,1)]$, are locally null rotation isotropic if and only if their curvature tensor and its first two derivatives are locally null rotation invariant.

As explained in Sect. 2.1.4, these spacetimes might have exhibited a sequence of $\left(s_{i}, t_{i}\right)$ values requiring a check of the fourth derivatives of the curvature, but the detailed calculations show that this is never the case.

Theorem 2 A spacetime other than those covered by Theorem 1 is locally null rotation isotropic if and only if the curvature tensor and its first derivative are locally null rotation invariant.

The LNRI condition in Theorem 2 is also sufficient in those subcases of the spacetime families covered by Theorem 1 for which LNRI of the first derivatives implies LNRI of the second derivatives (which does not alter the statement in Theorem 1). The specification of those subcases is given in Sections 3.1, 3.4, 3.5 and 3.6.

All locally null rotation isotropic spacetimes admit a multiply-transitive $G_{r}(r \geq 3)$.

Acknowledgements I am grateful to Jan Åman for his work in developing the software CLASSI and for discussions of the Petrov type N cases, and to Filipe Mena, both for the stimulus to this work given by our joint study of discrete isotropies and for his very careful reading of the first draft of this paper and useful suggestions for its improvement. I am also grateful to Charles Torre for correction of an error in that draft, to Graham Hall for comments and to two anonymous reviewers for comments and suggestions which enabled an improved presentation of the results.

\section{Declarations}

Conflict of interest The author is an Editorial Board member of the journal General Relativity and Gravitation.

Open Access This article is licensed under a Creative Commons Attribution 4.0 International License, which permits use, sharing, adaptation, distribution and reproduction in any medium or format, as long as you give appropriate credit to the original author(s) and the source, provide a link to the Creative Commons licence, and indicate if changes were made. The images or other third party material in this article are included in the article's Creative Commons licence, unless indicated otherwise in a credit line to the material. If material is not included in the article's Creative Commons licence and your intended use is not permitted by statutory regulation or exceeds the permitted use, you will need to obtain permission directly from the copyright holder. To view a copy of this licence, visit http://creativecommons.org/licenses/by/4.0/.

\section{A Known spacetimes with null rotation invariance}

Local invariance groups $g$ containing both a null rotation and either rotational or boost symmetry are possible only in conformally flat spaces with Ricci tensors of Segre types $[(11,2)]$ or $[1(11,1)]$ or $[(111,1)]$. Such spacetimes were discussed in the previous papers $[11,12]$. So the only metrics to be considered in this paper are those where $g$ consists solely of null rotations. 
Spacetimes with a two-dimensional LNRI group have at least a $G_{5}$ on an $N_{3}$. These are included in the $p p$-waves discussed below. Spacetimes with a one-dimensional LNRI group must have a group of motions $G_{r}$ with $r \geq 3$. If $r=3$ the group acts on a two-dimensional null surface $N_{2}$. If $r=4$ it acts on a three-dimensional hypersurface which may be null $\left(N_{3}\right)$ or timelike $\left(T_{3}\right)$. If $r \geq 5$ but $s=1$ the group acts on the whole spacetime. Groups $G_{6}$ or larger which contain null rotation isotropy necessarily act on the whole spacetime.

The $p p$ waves have the metric form

$$
\mathrm{d} a^{2}=2\left[-\mathrm{d} u \mathrm{~d} v-H \mathrm{~d} u^{2}+\mathrm{d} \xi \mathrm{d} \bar{\xi}\right], \quad H=H(\xi, \bar{\xi}, u)
$$

which is conformally flat only if $H_{, \bar{\xi} \bar{\xi}}=0$. Of the energy-momenta considered in [19] only vacuum and pure radiation (including Einstein-Maxwell) solutions are possible, with $\Lambda=0$. If $t_{p}=0$ the solutions are plane waves, which are special cases of (A.1), and there is a group $G_{6}$ acting transitively on spacetime, or in even more special cases with additional symmetry, a $G_{7}$ (see (24.46) and Section 12.2 in [19]).

The plane waves have $s=2$. Expressing this in terms of transitivity on the set of possible wave surfaces enabled Hall $[5,6]$ to show the equivalence of two definitions of the plane wave subcases of (A.1). He also proved that transitivity on the wave surfaces leads back to the metric (A.9) or those plane waves found in Sections 3.1, 3.4 and 3.5 above. (See the cited papers for the detailed assumptions made.)

The metric (A.1) has at least a group $G_{1}$ generated by $\boldsymbol{k}=\partial_{v}$, but may admit a $G_{r}$ with $r=2$ to 7 . The possible symmetry classes were studied in [18], without the use of the Einstein field equations. Those which have LNRI have $\left(s, t_{p}\right)=(2,1)$ and a $G_{5}$, or $\left(s, t_{p}\right)=(2,0)$ and a $G_{6}$, or $s=1$. There are none with a maximal $G_{4}$ so $\left(s, t_{p}\right)=(1,1)$ does not arise. If $\left(s, t_{p}\right)=(1,2)$, there is a $G_{3}$ on $N_{2}$ so these are included in (A.2) below. If $\left(s, t_{p}\right)=(1,0)$ there is a $G_{5}$.

The $p p$-waves with a $G_{5}$ or $G_{6}$ are listed as cases 9 to 15 in Table II in [18]. Case 9 is a homogeneous pure radiation spacetime, (12.36) in [19]. Case 10 is the only metric with a $G_{5}$ on null hyperplanes and $t_{p}=1$ : it is interpretable as an Einstein-Maxwell plane wave. Of those with a $G_{6}$, cases $11-14$ are pure radiation plane wave metrics, while case 15 is a vacuum solution of Einstein's equations. The formulae for these cases appear in the first paragraph of page 386 of [19], where $\kappa$ may be zero.

Note that despite claims to the contrary in the literature, repeated in [19] Section 24.5 , all these $p p$-wave metrics with LNRI admit interpretations as Einstein-Maxwell spacetimes, although in one case (that given as (24.47) or equivalently (12.36) in [19]) the Maxwell field does not inherit the spacetime's symmetries [20].

The general metric with a $G_{3}$ on $N_{2}$ was given by Barnes [1], who showed that it was equivalent to metric forms used earlier by Petrov and by Defrise. It can be written

$$
\mathrm{d} s^{2}=B^{2}(x, u)\left[-2 \mathrm{~d} u \mathrm{~d} v+2 F(x, u) \mathrm{d} u^{2}+\mathrm{d} y^{2}\right]+\mathrm{d} x^{2} .
$$

This has as (possibly) non-zero entries in $\Phi_{A B^{\prime}}$ exactly the components allowed in the canonical forms compatible with LNRI in which $\Phi_{A B^{\prime}}$ is not identically zero. In the null tetrad $\{B(\mathrm{~d} v-F \mathrm{~d} u), B \mathrm{~d} u,(d x-i B \mathrm{~d} y) / \sqrt{2},(\mathrm{~d} x+i B \mathrm{~d} y) / \sqrt{2}\}$ the curvature components are 


$$
\begin{aligned}
\Psi_{4} & =-\frac{1}{2}\left(F_{, x x}+F_{, x} B_{, x} / B-B_{, u u} / B^{3}+2\left(B_{, u}\right)^{2} / B^{4}\right), \\
\Phi_{02^{\prime}} & =2 \Phi_{11^{\prime}}=-\frac{1}{2}\left(B B_{, x x}-\left(B_{, x}\right)^{2}\right) / B^{2} \\
\Phi_{12^{\prime}} & =-\left(B B_{, u x}-B_{, u} B_{, x}\right) / \sqrt{2} B^{3} \\
\Phi_{22^{\prime}} & =-\frac{1}{2}\left(F_{, x x}+3 F_{, x} B_{, x} / B+B_{, u u} / B^{3}-2\left(B_{, u}\right)^{2} / B^{4}\right), \\
\Lambda & =-\frac{1}{4}\left(B B_{, x x}+\left(B_{, x}\right)^{2}\right) / B^{2} .
\end{aligned}
$$

Boosting from this frame to the frame with $\left|\Psi_{4}\right|=1$ used in the main text would in general lead to more complicated formulae. The $G_{3}$ is in general maximal but there are cases with a $G_{r}, r>3$, such as $p p$-waves, and spacetimes with a $G_{4}$ on $T_{3}$ [8].

Only if $B_{, x}=0$ can one have $\Phi_{11^{\prime}}=\Phi_{02^{\prime}}=\Lambda=0$ in the metric (A.2), and then $\Phi_{12^{\prime}}=0$ and $\Phi_{22^{\prime}}+\Psi_{4}=0 . \Phi_{12^{\prime}}=0$ if and only if $B$ is separable, taking the form $B_{1}(u) B_{2}(x)$. If $\Phi_{11^{\prime}}=\Phi_{02^{\prime}}=0$ then $B=Y(u) e^{K(u) x}$ where $Y(u)$ and $K(u)$ are functions of $u$ alone; then $\Lambda=-K^{2} / 2$. If $\Lambda=0$ then $B$ has the form $Y(u) \sqrt{x+K(u)}, Y$ and $K$ again being functions of $u$ alone. Spacetimes obeying one of $\Phi_{11^{\prime}}=0, \Phi_{12^{\prime}}=0$ or $\Lambda=0$, with suitable $F$, may also obey $\Phi_{22^{\prime}}=0$ or $\Psi_{4}=0$.

Barnes considered the Einstein equations for (A.2) for a Ricci tensor of Segre type [(112)] ("pure radiation") with $\Lambda=0$ and showed that all such spacetimes are $p p$ waves; they admit a Maxwell field that inherits the LNRI symmetry only in the more special plane wave case (cf. Sect. 3.4 above).

Barnes also found an Einstein space with the $B=Y(u) e^{K(u) x}$ form where $K=C$, a constant, $Y=1 / u$ and $F=G(u) e^{-3 C x}$. The existence of this Einstein space and others mentioned below shows that the assertion of [16] that nontwisting type $\mathrm{N}$ spacetimes with a cosmological constant admit at most a $G_{2}$ of isometries is mistaken. There are further Petrov type N Einstein spaces of the form (A.2) which Barnes overlooked. In these, $K=C$ and $F=G(u) e^{-3 C x}$ again, but $Y(u)$ is constant, say 1. If $G(u)$ is constant this is Kaigorodov's solution ((12.34) and (38.2) in [19]), up to constant scale factors, and otherwise is the $H=A(u) x^{3}$ solution for (A.8) below.

One can find further metrics for other Segre type, Weyl tensor, and $\Lambda$ combinations, though these are generally of less physical significance. Some examples are, by Segre type:

$[(1,3)]$, Petrov N, $\Lambda \neq 0 ; B=u^{x-1}, F=1$.

$[1(1,2)]$, Petrov N, $\Lambda \neq 0 ; B=u x, F=1$.

$[1(1,2)]$, conformally flat, $\Lambda=0 ; B=\sqrt{x} / u, F=\sqrt{x}$.

$[(11,2)]$, Petrov N, $\Lambda \neq 0 ; B=Y(u) e^{C x}, F=1$.

$[1(11,1)]$, Petrov N, $\Lambda=0 ; B=\sqrt{x} / u, F=1 / \sqrt{x}$.

Siklos [17] introduced the class of Lobatchevski plane waves, which include the only Einstein spaces conformal to $p p$-waves. The metrics can be written (omitting a constant factor fixing an overall scale for the curvature) as

$$
\mathrm{d} s^{2}=\left[-2 \mathrm{~d} u \mathrm{~d} v-H \mathrm{~d} u^{2}+\left(\mathrm{d} x^{2}+\mathrm{d} y^{2}\right)\right] / x^{2}, \quad H=H(x, y, u) .
$$

They are of Petrov type $\mathrm{N}$ and have in general a pure radiation energy-momentum tensor (Segre type $[(11,2)]$ ), a constant negative $\Lambda$ (so $p p$-waves are not possible), and in general only a $G_{1}$ of isometries generated by $\partial_{v}$. Particular cases may admit 
a $G_{r}, 2 \leq r \leq 6$ : the special $H$ admitting these larger groups, and their associated Killing vectors, are listed in Table 1 of Siklos' paper. From that table, there is a oneparameter LNRI group only if $H_{, y}=0$. Under that condition the spacetime admits a $G_{3}$ on $N_{2}$ in general, a $G_{4}$ on $T_{3}$ if $H$ is $A(x)$, a $G_{4}$ on $N_{3}$ if $H$ is $A(u) x^{2}$ or $u^{-2 b-2} A\left(x u^{b}\right)$ with constant $b$, a $G_{5}$ on $V_{4}$ if $H= \pm x^{a}$ for constant $a$ and a $G_{6}$ on $V_{4}$ if $H= \pm x^{-2}$. The last of these is Defrise's pure radiation solution with a cosmological constant, (12.6) in [19], i.e.

$$
\mathrm{d} s^{2}=\frac{3}{|\Lambda| y^{2}}\left[\mathrm{~d} y^{2}+\mathrm{d} z^{2}-\mathrm{d} v\left(\mathrm{~d} u-\frac{\Lambda \mathrm{d} v}{|\Lambda| y^{2}}\right)\right] .
$$

( $\Lambda$ here being the cosmological constant, not the Newman-Penrose $\Lambda$.) If $H=A(u) x^{3}$ in these cases the spacetime is an Einstein space, which when $A$ is constant is Kaigorodov's solution, (38.2) in [19]. The $G_{5}$ of the Kaigorodov solution is maximal for type N Einstein spaces.

The possible metrics for a $G_{4}$ acting on a null hypersurface $N_{3}$ (not necessarily with LNRI) were found by Kruchkovich and Petrov. The list given in [15], Section 32, has a few readily correctable misprints. It also includes two cases with non-Lorentzian signature, and one which (as noted by Petrov) does not allow a null hypersurface. Inspection of the remaining metrics shows that in most cases the isotropy in the given isometry group $G_{4}$ is a spatial rotation rather than a null rotation.

These remaining metrics were considered further by Lauten and Ray [7], who found no solutions of Einstein's equations for a range of possible energy-momenta in all but one case, but noted, without finding general-relativistic solutions, that three metrics admitted additional Killing vectors. Two of these they denote by $G_{4} V I_{2}$ and $G_{4} V I_{4}$ in their table (page 885) but $G_{4} V I_{1}$ and $G_{4} V I_{2}$ on page $886^{5}$. One of those, (32.26) in Petrov's book, where it is labelled $G_{4} V I_{3}$ and also appears as (33.1) labelled $G_{5} A$, admits a $G_{5}$ on $N_{3}$ and is a $p p$-wave in coordinates different from those of (A.1) above. The other, (32.27) or $G_{4} V I_{2}$ in the book, taking $e=1$ to give the correct signature, is a special case of (32.26) with plane symmetry.

Lauten and Ray's one relativistic solution is for Petrov's $G_{4} I_{1}$ metric, which they give with misprints corrected. It is a pure radiation solution with an arbitrary $\Phi_{22^{\prime}}$ dependent on their $\left(x_{1}-x_{4}\right)$ and $\Lambda \neq 0$ : they call it a null fluid with a cosmological constant. The metric is a special case of (A.2), as can be shown using the calculations in Barnes' paper, with an additional translational Killing vector in the null hyperplane $u=$ constant.

The possibilities for a group $G_{4}$ with LNRI acting on a $T_{3}$ were studied in [8], and are either special cases of (A.2) or have metrics of the form

$$
\mathrm{d} s^{2}=A^{2}(z)\left[-2 e^{y} \mathrm{~d} u\left(\mathrm{~d} v+B(z) e^{y} \mathrm{~d} u\right)+\mathrm{d} y^{2}\right]+\mathrm{d} z^{2},
$$

which appears as (13.19) in [19]. Its Ricci tensor has Segre type [1(1,2)] or one of its degeneracies, and it is conformally flat only if $B=0=\Phi_{22^{\prime}}$ (Segre type [1(11,1)]. In that case $\Phi_{11^{\prime}}=\left(1+4\left(A_{, z}^{2}-A A_{, z z}\right)\right) / 16 A^{2}$.

\footnotetext{
${ }^{5}$ A comparison of Petrov's and other enumerations of the possible structures of real $G_{4}$ can be found in [9].
} 
Finally one could have a homogeneous spacetime with a group $G_{5}$ acting transitively and including a null rotation isotropy, when $s=1$ and $t_{p}=0$. Such metrics are known for an Einstein space, for pure radiation, and for a combination of these (see (12.34), (12.36) and (12.38) in [19]) but these examples are special cases of the other metrics above.

One can thus anticipate that assuming $\left(\mathrm{A}_{m}\right)$ when $g$ contains a null rotation invariance will lead to spacetimes whose metrics can be put in one of the forms (A.1), (A.2), (A.8), or (A.10). They may have Petrov type $\mathrm{N}$ or be conformally flat, and the Ricci tensors can be: for (A.1), [(11,2)]; for (A.2) any of those types considered above; for Siklos, only $[(11,2)]$ with a non-zero $\Lambda$; and for $(A .10),[1(1,2)]$ or $[1(11,1)]$. However, with Segre type $[1(11,1)]$ where, unlike all those metrics, $\kappa$ need not be zero, there may be other possibilities.

\section{B The Bianchi identities under LNRI}

This appendix gives the Bianchi identities assuming, for the reasons discussed in Sect. 2.1 , that the only components of the curvature which might be non-zero are $\Psi_{4}$, which is constant and such that $\left|\Psi_{4}\right|=1, \Lambda$, and the real components $\Phi_{02^{\prime}}=\Phi_{11^{\prime}}, \Phi_{12^{\prime}}$ and $\Phi_{22^{\prime}}$.

In more detail, only the following components of $\Phi_{A B^{\prime}}$ remain in the allowed Segre types: in $[(1,3)], \Phi_{12^{\prime}}$; in [1(1,2)], $2 \Phi_{11^{\prime}}=\Phi_{02^{\prime}}$ and $\Phi_{22^{\prime}}$; in $[(11,2)], \Phi_{22^{\prime}}$; and in $[1(11,1)], 2 \Phi_{11^{\prime}}=\Phi_{02^{\prime}}$. Since $\Phi_{02^{\prime}}$, if non-zero, will be equal to $2 \Phi_{11^{\prime}}$ in the canonically chosen frames, that is assumed below.

For all curvature components, $D Q=0$, and $\delta Q=\bar{\delta} Q$ is real. Since under these conditions $(\mathrm{Be})$ reads $(\rho+\bar{\sigma}) \Phi_{11^{\prime}}=0$, that combination of terms has been eliminated from $(\mathrm{Bb})$ and $(\mathrm{Bi})$. The conditions (2.12) and (2.14) have been applied to terms involving $\Psi_{4}$.

$$
\begin{aligned}
& B a:-2(\kappa+\bar{\kappa}) \Phi_{11^{\prime}}=0, \\
& B b: 2(2 \varepsilon-2 \bar{\varepsilon}) \Phi_{11^{\prime}}-2 \kappa \Phi_{12^{\prime}}=0, \\
& B c: \delta \Phi_{12^{\prime}}-2 \Delta \Phi_{11^{\prime}}=5 \varepsilon \Psi_{4}+2(2 \gamma-2 \bar{\gamma}+\bar{\mu}+\lambda) \Phi_{11^{\prime}} \\
& \quad+2(\bar{\tau}-\alpha) \Phi_{12^{\prime}}-\bar{\sigma} \Phi_{22^{\prime}} \\
& B d: \delta \Phi_{22^{\prime}}-\Delta \Phi_{12^{\prime}}=(4 \beta-\tau) \Psi_{4}-2(v+\bar{v}) \Phi_{11^{\prime}} \\
& \quad+2(\gamma+\bar{\mu}+\lambda) \Phi_{12^{\prime}}+(\bar{\tau}-2 \bar{\beta}-2 \alpha) \Phi_{22^{\prime}} \\
& B f: 2 \Delta \Lambda-\delta \Phi_{12^{\prime}}=-2(\mu+\bar{\lambda}) \Phi_{11^{\prime}}+2(\pi+\bar{\pi}+\beta) \Phi_{12^{\prime}} \\
& \quad+(\bar{\rho}-2 \varepsilon-2 \bar{\varepsilon}) \Phi_{22^{\prime}} \\
& B g: 2 \delta \Phi_{11^{\prime}}-2 \delta \Lambda=2(2(\bar{\alpha}-\beta)-(\pi+\bar{\pi})) \Phi_{11^{\prime}} \\
& \quad-2(\bar{\rho}-\varepsilon) \Phi_{12^{\prime}}+\bar{\kappa} \Phi_{22^{\prime}} \\
& B h: 2 \delta \Phi_{11^{\prime}}-2 \delta \Lambda=2(2(\alpha-\bar{\beta})+(\tau+\bar{\tau})) \Phi_{11^{\prime}}-2 \rho \Phi_{12^{\prime}}, \\
& B i:(\kappa+\bar{\kappa}) \Phi_{12^{\prime}}=0, \\
& B j:-3 \delta \Phi_{11^{\prime}}+3 \delta \Lambda=2(-2 \alpha+2 \bar{\beta}+\pi+\bar{\pi}-\tau-\bar{\tau}) \Phi_{11^{\prime}}
\end{aligned}
$$




$$
\begin{aligned}
& +(\bar{\rho}+\sigma+2 \rho-2 \bar{\varepsilon}) \Phi_{12^{\prime}}-\kappa \Phi_{22^{\prime}} \\
& B k: 3 \Delta \Lambda-2 \delta \Phi_{12^{\prime}}+\Delta \Phi_{11^{\prime}}=(\rho+\bar{\rho}-2 \varepsilon-2 \bar{\varepsilon}) \Phi_{22^{\prime}}-2(\mu+\bar{\mu}+\lambda+\bar{\lambda}) \Phi_{11^{\prime}} \\
& +(2(\beta+\bar{\beta}+\pi+\bar{\pi})-\tau-\bar{\tau}) \Phi_{12^{\prime}}
\end{aligned}
$$

\section{References}

1. Barnes, A.: On space-times admitting a three-parameter isometry group with two-dimensional null orbits. J. Phys. A 12, 1493 (1979)

2. Defrise, L. (1969). Groupes d'isotropie et groupes de stabilité conforme dans les espaces lorentziens. PhD thesis, Université Libre de Bruxelles

3. Ellis, G.F.R.: Dynamics of pressure-free matter in general relativity. J. Math. Phys. 8, 1171 (1967)

4. Hall, G.S.: Symmetries and Curvature Structure in General Relativity. Lecture Notes in Physics, vol. 46. World Scientific, Singapore (2004)

5. Hall, G. S. (2015). Symmetries, orbits and isotropy in general relativity theory. In Sharif, M., editor, Proceedings of the International Conference on Relativistic Astrophysics, Lahore, February 2015, pages 62-76. Punjab University Press, Lahore

6. Hall, G.S.: Some remarks on wave solutions in general relativity theory. Arab. J. Math. 8, 171-181 (2019)

7. Lauten, W.T., III., Ray, J.R.: Investigations of space-times with four-parameter groups of motions acting on null hypersurfaces. J. Math. Phys. 18, 855 (1977)

8. MacCallum, M. A. H. (1980). Locally isotropic spacetimes with non-null homogeneous hypersurfaces. In Tipler, F.J., editor, Essays in general relativity (A festschrift for A.H. Taub), pp. 121-138. Academic Press, New York

9. MacCallum, M.A.H.: On the classification of the real four-dimensional Lie algebras. In: Harvey, A.L. (ed.) On Einstein's Path: Essays in honor of Engelbert Schucking, pp. 299-317. Springer, New York (1999)

10. MacCallum, M.A.H.: Totally symmetrized spinors and null rotation invariance. Class. Quant. Grav. 37, 195011 (2020)

11. MacCallum, M.A.H.: Spacetimes with continuous linear isotropies I: spatial rotations. Gen. Rel. Grav. 53, $57(2021)$

12. MacCallum, M.A.H.: Spacetimes with continuous local isotropies II: boosts. Gen. Rel. Grav. 53, 61 (2021)

13. MacCallum, M.A.H., Åman, J.E.: Algebraically independent $n$-th derivatives of the Riemannian curvature spinor in a general spacetime. Class. Quant. Grav. 3(6), 1133-41 (1986)

14. Milson, R. Pelavas, N: The curvature homogeneity bound for Lorentzian metrics. Int. J. Geom. Meth. in Mod. Phys. 06, 99 (2009)

15. Petrov, A. Z.: Einstein spaces. Pergamon Press, Oxford. Translation by R.F. Kelleher of Russian edition published by Fitzmatlit, Moscow (1961)

16. Salazar, I., H., García D., A., Plebański, J. F.: Symmetries of the nontwisting type-N solutions with cosmological constant. J. Math. Phys. 24(8), 2191-2196 (1983)

17. Siklos, S. T. C.: Lobatchevski plane gravitational waves. In MacCallum, M.A.H., editor, Galaxies, axisymmetric systems and relativity. Essays presented to W.B. Bonnor on his 65th birthday., pages 247-274. Cambridge University Press, Cambridge (1985)

18. Sippel, R., Goenner, H.: Symmetry classes of pp-waves. Gen. Rel. Grav. 18(12), 1229-43 (1986)

19. Stephani, H., Kramer, D., MacCallum, M.A.H., Hoenselaers, C.A., Herlt, E.: Exact solutions of Einstein's field equations, 2nd edn., p. 2009. Cambridge University Press, Cambridge, Corrected Paperback edition (2003)

20. Torre, C.G.: All homogeneous pure radiation spacetimes satisfy the Einstein-Maxwell equations. Class. Quantum Grav. 29, 077001 (2012)

Publisher's Note Springer Nature remains neutral with regard to jurisdictional claims in published maps and institutional affiliations. 